\title{
Blockade of the extracellular signal-regulated kinase pathway by U0126 attenuates neuronal damage following circulatory arrest
}

\author{
Deog-Gon Cho, MD \\ Matthew R. Mulloy, MDa \\ Paul A. Chang, BS ${ }^{a}$ \\ Mahlon D. Johnson, MD, $\mathrm{PhD}^{\mathrm{a}}$ \\ Alon S. Aharon, MD ${ }^{\mathrm{b}}$ \\ Trevor A. Robison, BS ${ }^{a}$ \\ Tamara L. Buckles, BS ${ }^{a}$ \\ Daniel W. Byrne, MS ${ }^{\mathrm{a}}$ \\ Davis C. Drinkwater, Jr, MD
}

From the Vanderbilt University Medical Center, Nashville, Tenn, ${ }^{\text {a }}$ and St Louis University Health Sciences Center, St Louis, Mo. ${ }^{\text {b }}$

Read at the Eighty-third Annual Meeting of The American Association for Thoracic Surgery, Boston, Mass, May 4-7, 2003.

Received for publication May 2, 2003; revisions requested Aug 29, 2003; accepted for publication Sept 29, 2003.

Address for reprints: Davis C. Drinkwater, Jr, MD, William S. Stoney Professor and Chairman, Department of Cardiac and Thoracic Surgery, Vanderbilt University Medical Center, 1161 21st Ave South 2986 TVC, Nashville, TN, 37232-5734 (E-mail: davis.drinkwater@vanderbilt.edu).

J Thorac Cardiovasc Surg 2004;127:1033-40 $0022-5223 / \$ 30.00$

Copyright $\odot 2004$ by The American Association for Thoracic Surgery

doi:10.1016/j.jtcvs.2003.09.038

Objectives: The extracellular signal-regulated kinase pathway of the mitogenactivated protein kinase signal transduction cascade has been implicated in the neuronal and endothelial dysfunction witnessed following cerebral ischemia-reperfusion injury. Extracellular signal-regulated kinase is activated by mitogen-activated protein kinase/extracellular signal-regulated kinase $1 / 2$. We evaluated the ability of a mitogen-activated protein kinase/extracellular signal-regulated kinase 1/2-specific inhibitor (U0126) to block extracellular signal-regulated kinase activation and mitigate ischemic neuronal damage in a model of deep hypothermic circulatory arrest.

Methods: Piglets underwent normal flow cardiopulmonary bypass (control, $n=4$ ), deep hypothermic circulatory arrest $(n=6)$, and deep hypothermic circulatory arrest with U0126 $(\mathrm{n}=5)$ at $20^{\circ} \mathrm{C}$ for 60 minutes. The deep hypothermic circulatory arrest with U0126 group was given $200 \mu \mathrm{g} / \mathrm{kg}$ of U0126 45 minutes prior to initiation of bypass followed by $100 \mu \mathrm{g} / \mathrm{kg}$ at reperfusion. Following 24 hours of post-cardiopulmonary bypass recovery, brains were harvested. Eleven distinct cortical regions were evaluated for neuronal damage using hematoxylin and eosin staining. A section of ischemic cortex was further evaluated by immunohistochemistry with rabbit polyclonal antibody against phosphorylated extracellular signal-regulated kinase $1 / 2$.

Results: The deep hypothermic circulatory arrest and deep hypothermic circulatory arrest with U0126 groups displayed diffuse ischemic changes. However, the deep hypothermic circulatory arrest with U0126 group possessed significantly lower neuronal damage scores in the right frontal watershed zone of cerebral cortex, basal ganglia, and thalamus $(P \leq .05)$ and an overall trend toward neuroprotection versus the deep hypothermic circulatory arrest group. This neuroprotection was accompanied by nearly complete blockade of phosphorylated extracellular signal-regulated kinase in the cerebral vascular endothelium.

Conclusions: In this experimental model of deep hypothermic circulatory arrest, U0126 blocked extracellular signal-regulated kinase activation and provided a significant neuroprotective effect. These results support targeting of the extracellular signal-regulated kinase pathway for inhibition as a novel therapeutic approach to mitigate neuronal damage following deep hypothermic circulatory arrest. 


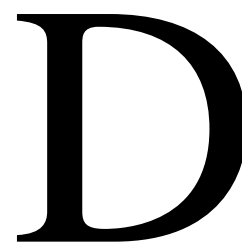

espite the known risk of neurologic sequelae, deep hypothermic circulatory arrest (DHCA) remains an important element in the repair of complex congenital heart defects. Although DHCA has contributed to the improvement of surgical results in neonates with congenital heart disease, little progress has been made in our ability to ameliorate the associated neurologic morbidity. Central nervous system sequelae such as seizures, neurocognitive deficits, developmental delay, and motor dysfunction occur in $5 \%$ to $45 \%$ of survivors. ${ }^{1,2}$ This unacceptably high rate of neurologic complications has stimulated the development of novel neuroprotective strategies for the prevention of neuronal damage in patients undergoing DHCA. ${ }^{3-7}$

Mitogen-activated protein kinases (MAPKs) represent a family of highly conserved serine/threonine protein enzyme complexes that are critical components of the enzyme cascade systems that connect a wide variety of cell surface receptors to critical cellular targets. ${ }^{8}$ MAPKs are activated during ischemia and/or reperfusion and contribute to the structural and functional changes associated with ischemiareperfusion (I/R) injury. ${ }^{9}$ Recent studies demonstrate that members of the MAPK signal transduction cascade, most notably extracellular signal-regulated kinase 1/2 (ERK1/2), c-Jun amino-terminal kinase (JNK), and p38 protein (p38), are activated following I/R in the brain, ${ }^{10,11}$ heart, ${ }^{12,13}$ kidney, ${ }^{14,15}$ and liver. ${ }^{16}$ The ERK1/2 subfamily, which is activated by MAPK/ERK kinase 1/2 (MEK1/2), has been heavily implicated in the neuronal and endothelial dysfunction witnessed following cerebral I/R injury. ${ }^{10}$ Pharmacologic studies with MEK1/2-specific inhibitors PD98059, ${ }^{17}$ 1,4-diamino-2,3-dicyano-1,4-bis[2-aminophenylthio]butadiene (U0126), ${ }^{18}$ and SL $327^{19}$ have shown that inhibition of the ERK1/2 pathway significantly reduces neuronal damage following cerebral ischemia and cerebral I/R injury.

The recognition that neuronal damage following cerebral $\mathrm{I} / \mathrm{R}$ injury may involve ERK1/2 activation has opened up a new and exciting opportunity for the pharmacologic modulation of cerebral tolerance to DHCA. Previous work by our group demonstrated that the ERK1/2 pathway is activated following DHCA and linked the expression of p-ERK1/2 within cerebral vascular endothelium to the postischemic neuronal damage seen following DHCA (unpublished data). The present study evaluated the ability of the MEK1/2-specific inhibitor (U0126) to block ERK1/2 activation and mitigate ischemic neuronal damage in a clinically applicable model of DHCA (Figure 1). Additionally, immunohistochemistry was performed to qualitatively correlate the presence of phosphorylated ERK1/2 (p-ERK1/2) expression in the cerebral vascular endothelium with the degree of neuronal damage present 24 hours following DHCA.

\section{Materials and Methods}

\section{Animals and Surgical Preparation}

Fifteen neonatal Duroc piglets with a mean body weight of $7.6 \mathrm{~kg}$ (range 5.6-10.3 kg) were divided into 3 groups: normal flow, normothermic cardiopulmonary bypass (CPB control, $\mathrm{n}=4$ ), DHCA (DHCA, $\mathrm{n}=6$ ), or DHCA with U0126 (DHCA $+\mathrm{U}, \mathrm{n}=$ 5). All animals received humane care in compliance with the Principles of Laboratory Animal Care formulated by the National Society for Medical Research and with the Guide for the Care and Use of Laboratory Animals prepared by the Institute of Laboratory Animal Resources and published by the National Institute of Health (NIH publication no. 85-23, revised in 1985). Piglets were sedated with intramuscular ketamine $(20 \mathrm{mg} / \mathrm{kg})$ and acepromazine $(0.03 \mathrm{mg} / \mathrm{kg})$, transported to the laboratory, and attached to a continuous pulse oximeter (Vet/Ox BP Plus 6500, Heska Co, Fort Collins, Colo). Inhalational anesthesia with $1.5 \%$ to $2.5 \%$ isofluorane was administered via a fitted, rubber-sealed mask. Once the animal reached a plane of surgical anesthesia as determined by toe-pinch response, jaw tone, heart rate, and response to stimulation, a rapid tracheostomy was performed with a $3.5-$ to $4.5-\mathrm{mm}$ endotracheal tube. The piglets were ventilated with an $\mathrm{FIO}_{2}$ of 1.0, tidal volumes of 10 to $15 \mathrm{cc} / \mathrm{kg}$, and a rate of 18 to 24 respirations per minute (Modified Servo Anesthesia-Ventilator 900C, Siemens Pediatric, Sweden) to achieve arterial oxygen tensions greater than $200 \mathrm{~mm} \mathrm{Hg}$ and carbon dioxide tensions of 35 to $45 \mathrm{~mm} \mathrm{Hg}$. Anesthesia was maintained by a continuous inhalation of $1.5 \%$ to $2.5 \%$ isofluorane throughout the entire experiment, except during the period of CPB when an isofluorane infusion was given through the bypass circuit. The right femoral artery and vein were cannulated for arterial pressure monitoring (Digital Display, Gould, Valley View, Ohio), collection of blood samples for blood gas analyses (Blood Gas Analyzer, Synthesis 25, Instrumentation Laboratory, Lexington, Mass), and administration of intravenous fluids. Prior to arterial cannulation, $300 \mathrm{IU} / \mathrm{kg}$ of heparin was administered via the femoral venous cannula. A rectal temperature probe (Yellow Springs Instrument Inc, Yellow Springs, Ohio) and suprapubic-indwelling bladder catheter were inserted. Following administration of cephazolin $(50 \mathrm{mg} / \mathrm{kg} \mathrm{IV})$ and a median sternotomy, bypass cannulas were placed into the ascending aorta (10F12F DLP, Medtronic Inc, Minneapolis, Minn), right atrium (16F20F DLP, Medtronic), and left atrium (10F DLP Vent Catheter, Medtronic). Rectal temperature was maintained at $37^{\circ} \mathrm{C}$ except for the period of induced hypothermia.

\section{Conduct of CPB}

The CPB circuit consisted of a Cobe nonpulsatile roller pump (Cobe Cardiovascular Inc, Arvada, Colo), cardiotomy reservoir, and hollow fiber membrane oxygenator (Minimax plus, Medtronic). The circuit was primed with fresh whole blood collected from an adult donor pig 1 day preoperatively, 2000 IU of heparin, $1 \mathrm{~g}$ of calcium chloride, and $25 \mathrm{mEq}$ of sodium bicarbonate. Hematocrit was maintained between $26 \%$ and $30 \%$. The total priming volume was 700 to $800 \mathrm{~mL}$. CPB was initiated at a flow rate of 100 to $150 \mathrm{~mL} / \mathrm{kg}$ per minute and adjusted to maintain a mean arterial pressure of 50 to $60 \mathrm{~mm} \mathrm{Hg}$.

Piglets were then subjected to 1 of the following conditions. In the $\mathrm{CPB}$ control group, piglets underwent normal flow (100 to 150 $\mathrm{mL} / \mathrm{kg} / \mathrm{min})$, normothermic $\left(37^{\circ} \mathrm{C}\right) \mathrm{CPB}$ for 60 minutes without 


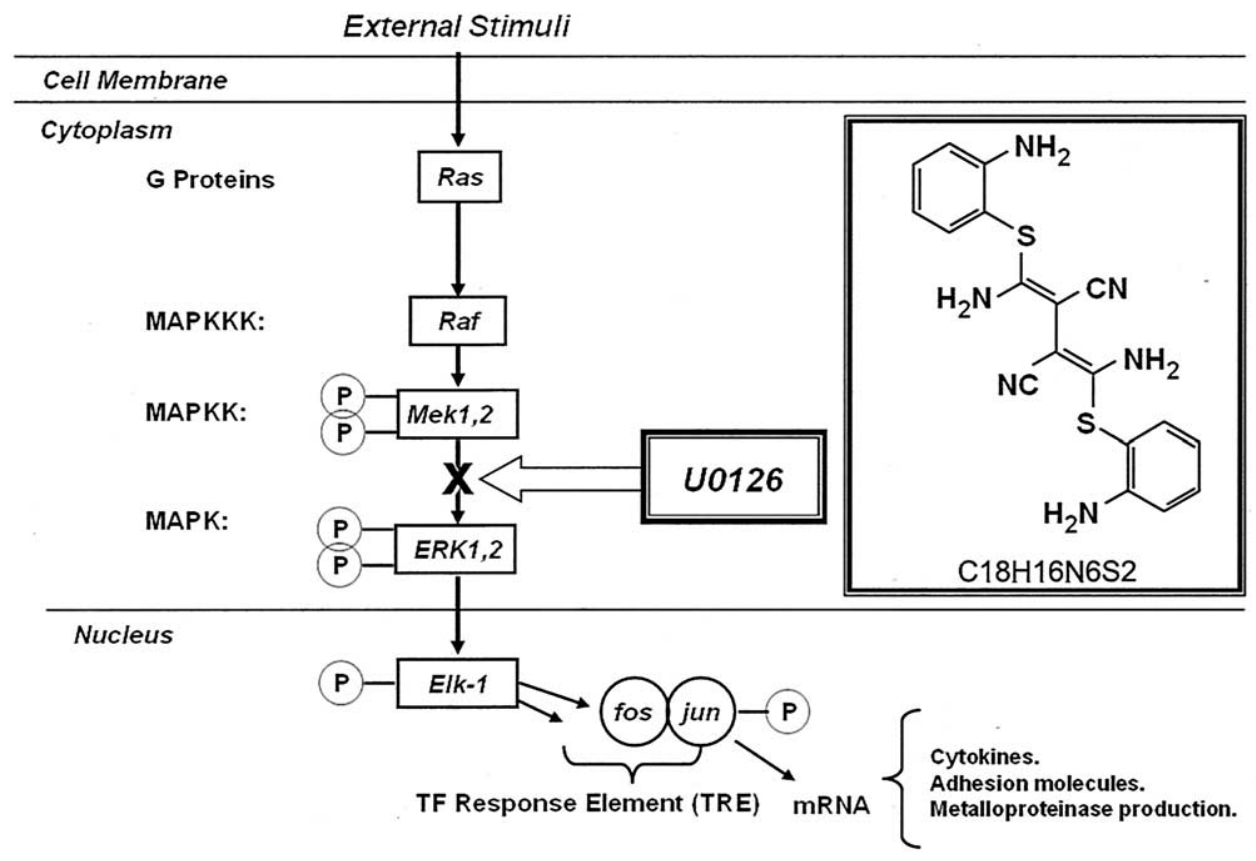

Figure 1. Mechanism of ERK1/2 cascade with specific MEK1/2 inhibitor U0126. ERK1/2, extracellular signalregulated kinases 1 and 2; MEK1/2, mitogen-activated protein kinase/extracellular signal-regulated kinase 1/2. Reprinted from Bioorganic \& Medicinal Chemistry Letters, Vol 8, Duncia JV, Santella JB 3rd, Higley CA, Pitts WJ, Wityak J, Frietze WE, et al. MEK inhibitors: the chemistry and biological activity of U0126, its analogs, and cyclization products. p 2839-44, copyright 1998, with permission from Elsevier.

aortic crossclamping. In the DHCA and DHCA + U groups, piglets were cooled to a rectal temperature of $20^{\circ} \mathrm{C}$ using a heat exchanger and cooling blanket. CPB was then discontinued and the piglets were maintained at $20^{\circ} \mathrm{C}$ for 60 minutes of DHCA. Myocardial protection was afforded by the infusion of high potassium blood cardioplegic solution $(15 \mathrm{~mL} / \mathrm{kg}$, potassium concentration $20 \mathrm{mEq}$ ) and application of iced saline slush during DHCA. Arterial blood gases were controlled using $\mathrm{pH}$ stat strategy. After 60 minutes, CPB was reinitiated and the piglets were warmed to a rectal temperature of $37^{\circ} \mathrm{C}$. Care was taken to avoid a temperature difference between the perfusate and core temperature of more than $8^{\circ} \mathrm{C}$. Once the piglets were normothermic they were weaned from CPB and decannulated. Protamine (1 mg/1000 IU heparin) was administered following separation from CPB.

\section{Administration}

U0126 (Calbiochem, La Jolla, Calif), a potent and specific inhibitor of MEK1/2, was administered via the femoral vein in the DHCA + U group (Figure 1). The piglets were given $200 \mu \mathrm{g} / \mathrm{kg}$ of U0126 45 minutes prior to initiation of CPB and $100 \mu \mathrm{g} / \mathrm{kg}$ at the time of reperfusion. The U0126 was dissolved in $0.25 \mathrm{~mL}$ of dimethylsulfoxide (Sigma Chemical Co, St Louis, Mo).

\section{Postoperative Management}

After weaning from $\mathrm{CPB}$, the animals remained intubated and sedated and were allowed to recover for 24 hours. Physiologic data were recorded every hour. To avoid postoperative adverse effect (bias), arterial blood gases, electrolytes, and hemodynamics were maintained within normal limits throughout the recovery period. Upon completion of the experiment, the cranial vault was opened and the fresh whole brain was transected at the level of $\mathrm{C} 1$ during deep anesthesia in a state of stable hemodynamics and continuous antegrade cerebral perfusion.

\section{Brain Histology and Neuronal Damage}

The resected brains were fixed in $10 \%$ neutral buffered formalin and stored at room temperature for 2 weeks. The brains were then sectioned coronally and examined. Sections were taken from the following sites: (1) right frontal watershed area, (2) left frontal watershed area, (3) right parietal watershed area, (4) left parietal watershed area, (5) right temporal lobe and hippocampus, (6) left temporal lobe and hippocampus, (7) basal ganglia, (8) anterior thalamus, (9) cerebellum, (10) mesencephalon, (11) pons, and (12) medulla oblongata. The tissue was processed overnight and subsequently embedded in paraffin. Five-micron sections were cut on a microtome, deparaffinized, and then stained with hematoxylin and eosin. A single neuropathologist (M.J.) blinded to the experimental group examined all specimens and scored the extent of neuronal damage in each slide. A modified grading system of Pulsinelli and colleagues ${ }^{20}$ was used to quantify neuronal damage. Scores were assigned based on the following scale: $0=$ no neuronal damage; $1=$ mild neuronal damage (rare or scattered ischemic neurons or a section with scattered neurons and 1 collection less than one $100 \times$ magnification field); $2=$ moderate neuronal damage (scattered collections of ischemic neurons that collectively occupy one to three $100 \times$ magnification fields); $3=$ 
TABLE 1. Intraoperative CPB profiles

\begin{tabular}{lccc}
\hline & $\begin{array}{c}\text { CPB control } \\
(\mathbf{n}=\mathbf{4})\end{array}$ & $\begin{array}{c}\text { DHCA } \\
(\mathbf{n}=\mathbf{6})\end{array}$ & $\begin{array}{c}\text { DHCA + U } \\
(\mathbf{n}=\mathbf{5})\end{array}$ \\
\hline $\mathrm{TCPB}(\mathrm{min})$ & $63.3 \pm 1.9^{*} \dagger$ & $87.0 \pm 9.9$ & $77.0 \pm 10.2$ \\
$\mathrm{ACC}(\mathrm{min})$ & - & $69.7 \pm 3.8$ & $71.8 \pm 3.3$ \\
$\mathrm{CA}(\mathrm{min})$ & - & 60 & 60 \\
$\mathrm{Hematocrit}(\%)^{*}$ & $29.7 \pm 0.6$ & $27.6 \pm 1.3$ & $26.6 \pm 1.5$ \\
$\mathrm{pH}^{*}$ & $7.45 \pm 0.05$ & $7.41 \pm 0.02$ & $7.39 \pm 0.04$ \\
$\mathrm{PaO}_{2}(\mathrm{~mm} \mathrm{Hg})^{*}$ & $307.3 \pm 14.7$ & $328.3 \pm 21.1$ & $269.0 \pm 30.9$ \\
$\mathrm{PaCO}_{2}(\mathrm{~mm} \mathrm{Hg})^{*}$ & $38.5 \pm 2.6$ & $36.2 \pm 2.2$ & $41.4 \pm 1.9$ \\
$\mathrm{MAP}_{(\mathrm{mm} \mathrm{Hg})}{ }^{*}$ & $59.3 \pm 1.7$ & $57.6 \pm 2.5$ & $56.6 \pm 3.1$ \\
Cooling time (min) & - & $32.8 \pm 2.9$ & $27.4 \pm 2.2$ \\
Rewarming (min) & - & $40.0 \pm 4.9$ & $46.0 \pm 4.7$ \\
\hline
\end{tabular}

$C P B$, Cardiopulmonary bypass; $D H C A$, deep hypothermic circulatory arrest; $D H C A+U$, deep hypothermic circulatory arrest with U0126; TCPB, total CPB time (not including DHCA time); $A C C$, aortic crossclamping time; $p H$, arterial pH; $\mathrm{PaCO}_{2}$, arterial partial pressure of carbon dioxide; $\mathrm{PaO}_{2}$, arterial partial pressure of oxygen; $M A P$, mean systemic arterial pressure. Values are shown as mean $\pm \mathrm{SE}$.

*Variables were measured at the midpoint of total CPB time for the CPB control group and approximately 10 minutes prior to DHCA for the other groups.

$\mathrm{\dagger} P<.05$, significant difference from the DHCA and DHCA $+\mathrm{U}$ groups by Kruskal-Wallis test.

severe neuronal damage (ischemic collections of neurons that collectively occupy four or more $100 \times$ magnification fields). Histopathologic scores for each group represented the average among the slides taken from each region. Ischemia was defined as nuclear pyknosis, eosinophilia, and contraction of cytoplasm seen in neurons in a high-powered field $(100 \times)$.

\section{Immunohistochemistry for Phosphorylated ERK1/2}

After 2 weeks of fixation, a section of the right frontal watershed zone (WSZ) was examined by immunohistochemistry using polyclonal rabbit antibodies to p-ERK1/2 and total ERK1/2 (Cell Signaling, Beverly, Mass). Immunohistochemistry was performed on a Ventana ES automated immunostainer (Ventana, Tucson, Ariz). Optimal conditions were determined separately for each antibody. To avoid background and artifactual unmasking of epitopes, no pretreatment was used. The antigen-antibody complexes were visualized using an avidin-biotin complex detection system (Vector Laboratories, Inc, Burlingame, Calif) with 3,3'diaminobenzidine as the chromogen. Sections were counterstained with hematoxylin. No significant immunoreactivity for each antibody was observed when substituting the primary antibodies with nonimmune rabbit serum or by skipping the primary antibodies. The activation of ERK1/2 was assessed by the same blinded neuropathologist (M.J.) as either nonsignificant (no or rare expression of p-ERK1/2) or significant (considerable positive expression of p-ERK1/2).

\section{Statistical Analysis}

Values are presented as the mean \pm standard error of the mean. The continuous variables were compared using an analysis of variance. A general linear model repeated measures analysis of variance was also employed to assess changes among groups from baseline to 6,12 , and 24 hours postoperatively. Differences in neuronal damage among the experimental groups were evaluated using Kruskal-Wallis test. A univariate general linear model was used to assess neuronal damage among groups after adjusting for covariates. Statistical analyses were conducted with the SPSS 11.5 software package (SPSS Inc, Chicago, Ill).

\section{Results}

\section{Comparison of Experimental Groups}

A comparison of physiologic variables at baseline and 6, 12, and 24 hours postoperatively (body weight, $\mathrm{pH}, \mathrm{PaCO}_{2}$, $\mathrm{PaO}_{2}$, hematocrit, $\mathrm{Na}, \mathrm{K}, \mathrm{Ca}^{2+}, \mathrm{HCO}_{3}{ }^{-}$, mean arterial blood pressure, and $\mathrm{FIO}_{2}$ ) revealed only the $\mathrm{PaO}_{2}$ at 12 hours postoperatively in the DHCA group to be a statistically important variable $(P=.033)$. The median total CPB time excluding DHCA was significantly longer in the DHCA group (87 minutes) and DHCA $+\mathrm{U}$ group (77 minutes) versus the $\mathrm{CPB}$ control group (63 minutes). However, there were no significant differences in intraoperative variables between the DHCA and DHCA + U groups (Table 1).

\section{Histologic Evaluation of Neuronal Damage}

Neuronal damage in circulatory arrest. The quantitative scores of neuronal damage for each area are displayed in Table 2. The CPB control group demonstrated nonsignificant neuronal damage in all areas. In contrast, piglets in the DHCA group demonstrated significant neuronal damage in the right and left frontal WSZs, right parietal WSZ, basal ganglia, thalamus, and cerebellum (Figure 2). The neuronal damage scores from the DHCA group were moderate to severe in the $4 \mathrm{WSZs}$ and mild to moderate in the hippocampus, basal ganglia, thalamus, cerebellum, mesencephalon, and pons.

Neuronal damage in circulatory arrest with U0126. In comparison with the CPB control group, the DHCA $+\mathrm{U}$ group demonstrated a more diffuse pattern of postischemic neuronal changes. However, there were no statistically significant differences in neuronal damage scores between the 2 groups (Table 2). The DHCA $+U$ group possessed significantly lower neuronal damage scores in the right frontal WSZ $(P=.009)$, basal ganglia $(P=.05)$, and thalamus $(P=.05)$ and an overall trend toward neuroprotection versus the DHCA group (Figure 2). The significant differences in neuronal damage seen in the right frontal WSZ, basal ganglia, and thalamus were unchanged even when covariates, such as the value for $\mathrm{PaO}_{2}$ at 12 hours in the DHCA group, were taken into account.

Activation of ERK1/2. Immunohistochemistry staining revealed significant expression of p-ERK1/2 in the cerebral vascular endothelium in 3 of the 6 DHCA piglets at 24 hours postoperatively. In contrast, nonsignificant expression of p-ERK1/2 appeared in the cerebral vascular endothelium of animals from the CPB control and DHCA + U groups. 


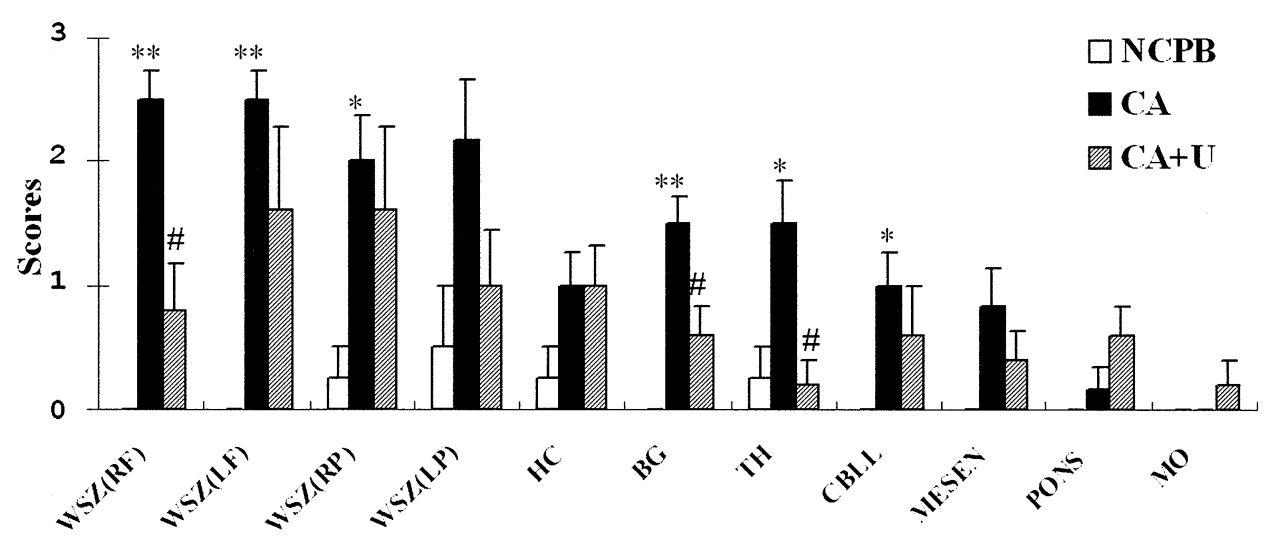

Brain Regions

Figure 2. The average ischemic neuronal damage score by experimental groups and by regions of the piglet brains. Data are shown as mean \pm SE. ${ }^{*} P<.05,{ }^{*} P<.01$ versus NCPB; significantly greater neuronal damage in the CA group. $\# P<.05$ versus $C A$; significantly less neuronal damage in the $C A+U$ group. $N C P B$, cardiopulmonary bypass control group; $C A$, deep hypothermic circulatory arrest group; $C A+U$, deep hypothermic circulatory arrest + U0126; WSZ, watershed zone of cerebral cortex; $R F$, right frontal; $L F$, left frontal; $R P$, right parietal; $L P$, left parietal; HC, hippocampus; BG, basal ganglia; $T H$, thalamus; CBLL, cerebellum; MESEN, mesencephalon; MO, medulla oblongata.

TABLE 2. Quantitative analysis of neuronal damage

\begin{tabular}{|c|c|c|c|c|c|}
\hline Site & $\begin{array}{c}\text { NCPB } \\
(n=4)\end{array}$ & $\begin{array}{c}\text { DHCA } \\
(n=6)\end{array}$ & $\begin{array}{c}\text { DHCA + U } \\
(n=5)\end{array}$ & $\begin{array}{c}P \text { value, } \\
\text { NCPB vs } \\
\text { DHCA }\end{array}$ & $\begin{array}{c}P \text { value, } \\
\text { DHCA vs } \\
\text { DHCA + U }\end{array}$ \\
\hline Right frontal WSZ & 0 & $2.5 \pm 0.22$ & $0.8 \pm 0.37$ & .010 & .009 \\
\hline Left frontal WSZ & 0 & $2.5 \pm 0.22$ & $1.6 \pm 0.68$ & .010 & .329 \\
\hline Right parietal WSZ & $0.25 \pm 0.25$ & $2.0 \pm 0.37$ & $1.6 \pm 0.68$ & .019 & .537 \\
\hline Left parietal WSZ & $0.5 \pm 0.5$ & $2.17 \pm 0.48$ & $1.0 \pm 0.45$ & .067 & .126 \\
\hline Hippocampus & $0.25 \pm 0.25$ & $1.0 \pm 0.26$ & $1.0 \pm 0.32$ & .114 & .99 \\
\hline Basal ganglia & 0 & $1.5 \pm 0.22$ & $0.6 \pm 0.24$ & .010 & .05 \\
\hline Thalamus & $0.25 \pm 0.25$ & $1.5 \pm 0.34$ & $0.2 \pm 0.2$ & .067 & .05 \\
\hline Cerebellum & 0 & $1.0 \pm 0.26$ & $0.6 \pm 0.4$ & .038 & .429 \\
\hline Mescencephalon & 0 & $0.83 \pm 0.31$ & $0.4 \pm 0.24$ & .114 & .429 \\
\hline Pons & 0 & $0.17 \pm 0.17$ & $0.6 \pm 0.24$ & .762 & .247 \\
\hline Medulla oblongata & 0 & 0 & $0.2 \pm 0.2$ & .99 & .662 \\
\hline
\end{tabular}

$N C P B$, Normothermic cardiopulmonary bypass control; $D H C A$, deep hypothermic circulatory arrest; $D H C A+U$, deep hypothermic circulatory arrest with U0126; WSZ, watershed zone of cerebral cortex. Values are shown as mean \pm SE. Score range $0-3$, representing no neuronal damage (0) and severe damage (3). $P$ values are by Mann-Whitney $U$ test.

\section{Discussion}

The MAPK signal transduction cascade has been found to play a key role in the process of I/R injury in a variety of organ systems. ${ }^{10-16}$ The ERK1/2 pathway of the MAPK cascade has emerged as a key mediator of cerebral I/R injury. ${ }^{10}$ Inhibition of this pathway in models of cerebral ischemia and cerebral I/R injury provided a significant neuroprotective effect. ${ }^{10,17,18}$ Additionally, our group has previously established that the ERK1/2 pathway is activated following DHCA and that an association exists between the expression of p-ERK1/2 in the cerebral vascular endothelium and the degree of neuronal damage present 24 hours following DHCA (unpublished data). Our findings implicated ERK1/2 activation in the pathogenesis of the postischemic neuronal damage seen following DHCA for the first time and established the validity of our model for use in the study of the cerebral I/R injury associated with DHCA. In the present study we examined the ability of an MEK1/ 2-specific inhibitor (U0126) to block the expression of p-ERK1/2 in the cerebral vascular endothelium and decrease ischemic neuronal damage following DHCA.

U0126 is a potent and highly specific inhibitor of MEK1/2, the upstream kinase of the ERK1/2 molecule, which noncompetitively binds the MEK1/2 molecule and 
prevents its phosphorylation and activation. ${ }^{21,22}$ The use of U0126 blocks the ability of MEK1/2 to phosphorylate and activate the ERK1/2 molecule and the pathway is rendered inactive at this point (Figure 1). U0126 inhibits ERK1/2 activation in a dose-dependent manner both in vitro ${ }^{21}$ and in vivo in ischemic cortical neurons. ${ }^{18}$ In our study, the U0126 dosing regimen was based upon the findings of Namura and colleagues, ${ }^{18}$ who demonstrated maximal cerebral protection with a $200-\mu \mathrm{g} / \mathrm{kg}$ intravenous pretreatment dose of U0126, which resulted in a $42 \%$ reduction in infarction volume in a rat stroke model. Based on these findings we administered a $200-\mu \mathrm{g} / \mathrm{kg}$ dose of U0126 45 minutes prior to $\mathrm{CPB}$ in an attempt to achieve maximal neuronal protection in our model. Additionally, a half dose of U0126 was given at the time of reperfusion in an attempt to lessen the acute effects of the cerebral reperfusion injury. Using this regimen we obtained nearly complete blockade of ERK1/2 activation in the cerebral vascular endothelium. In preliminary studies by our group, U0126 pretreatment given 10 minutes before DHCA was unable to adequately block ERK1/2 activation (data not shown). However, when U0126 was administered at this time the piglet was on CPB. As a result, the dose of U0126 was diluted by the volume of the CPB prime $(700 \mathrm{~mL})$. We believe that not only did these piglets not receive an adequate dose of U0126, but that 10 minutes was not an adequate amount of time for pretreatment of the brain. In contrast, when given 45 minutes prior to the initiation of CPB, the dose of U0126 was not affected by the volume of the priming solution and there was adequate time for pretreatment of the piglet brain. These results suggest that both the timing of U0126 administration and the concentration administered are essential factors in the production of an adequate blockade of ERK1/2 activation.

In the current study, an extensive assessment of the neuronal damage present 24 hours following normothermic $\mathrm{CPB}$, DHCA, and DHCA + U was performed by a single, blinded neuropathologist. Although the DHCA + U group displayed diffuse ischemic changes, the ischemic damage was not significant when compared with the normothermic CPB control group (Figure 2). The treatment of the DHCA + U group with U0126 resulted in significantly lower neuronal damage scores in the right frontal WSZ, basal ganglia, and thalamus and an overall trend toward neuroprotection versus the DHCA group (Figure 2). These results represent the first report of the ability of an inhibitor of the ERK1/2 pathway to attenuate neuronal damage following DHCA. In addition to our neuropathological analysis, sections of the right frontal WSZ were analyzed with immunohistochemistry. All specimens from the normothermic $\mathrm{CPB}$ control and the DHCA + U groups were found to have nonsignificant expression of p-ERK1/2 within the cerebral vascular endothelium. In contrast, 3 of the 6 specimens from the DHCA group possessed significant p-ERK1/2 expres- sion. These findings suggest that the ERK1/2 pathway serves as a key mediator of the cerebral I/R injury associated with DHCA and further demonstrate the ability of U0126 to effectively block the activation of the ERK1/2 pathway in our model of DHCA. Overall, the present study supports the selective targeting of the ERK1/2 pathway for inhibition as a means of increasing cerebral tolerance to DHCA and opens up several novel areas for future research.

\section{Limitations of the Study}

Prior to discussing the implications of our results, it is important to address 2 important methodological aspects of our study. First, the timing of the removal of the piglet brains following DHCA was a critical element within this study. Previous work has shown that following cerebral I/R injury a minimum of 24 hours is required before an accurate neuropathological assessment of the extent of neuronal damage can be made. ${ }^{20}$ However, the peak time for ERK1/2 activation following cerebral I/R injury has been shown to occur approximately 2 hours following reperfusion. ${ }^{10}$ Thus, while the optimal time for analysis of cerebral vascular endothelial ERK1/2 activation would have been prior to 24 hours, we chose to perform our analysis at this time to ensure an accurate neuropathologic assessment of the effectiveness of the U0126 molecule as an MEK1/2 inhibitor within our model of DHCA.

Second, we did not utilize the perfusion-fixation method of cerebral extraction. However, our method of rapid extraction ( $<5$ minutes) under a state of stable hemodynamics with continuous antegrade cerebral perfusion was found to be reliable and safe. Analysis of the brains from the CPB control group revealed no significant neuronal injury or expression of $\mathrm{p}$-ERK1/2. These findings demonstrate that our method of extraction has not introduced experimental error or bias into our findings.

\section{Interpretation of Results}

Although our pathologic and immunohistochemistry data clearly demonstrated a significant neuroprotective effect of U0126 following DHCA, a considerable degree of neuronal damage remained in the DHCA + U group despite its use. This suggests that the neuroprotective effect of U0126 may result from a partial inhibition of the ERK1/2 pathway or the complete inhibition of the ERK1/2 pathway circumvented in some manner by an unknown mechanism of "cross talk" involving the other MAPK pathways (p-38 and JNK). Additionally, the effects of other inflammatory cascades, such as the nuclear factor kappa beta cascade, were not accounted for by this study and may be responsible for the remaining neuronal damage seen in the DHCA $+\mathrm{U}$ group. Therefore, future studies are required to elucidate the exact mechanism of the neuroprotective effect of U0126 and to identify the cross talk that exists between the various 
pathways of the MAPK cascade and between the MAPK cascade and other proinflammatory cascades.

Although the results of our immunohistochemical analyses are encouraging, they must be cautiously interpreted. These results represent the initial evidence of a link between the presence of cerebral vascular endothelial expression of p-ERK $1 / 2$ and a more extensive pattern of neuronal damage 24 hours following DHCA. However, we recognize that the significance of such results must be weighed against 2 important factors. Initially, it must be understood that such immunohistochemical analyses yield qualitative and not quantitative results. Secondly, as previously stated, following cerebral I/R injury the peak activation of ERK1/2 has been shown to occur 2 hours following reperfusion. Therefore, the fact that only 3 of the 6 piglets demonstrated significant p-ERK1/2 expression in the cerebral vascular endothelium 24 hours after DHCA was likely due to the timing of cerebral extraction. To establish a more concrete correlation between the cerebral vascular expression pattern of p-ERK $1 / 2$ and the extent of neuronal damage seen following DHCA, further studies are required to examine the early activation pattern of the ERK1/2 pathway following DHCA. Ongoing studies in our laboratory are aimed at qualitatively and quantitatively assessing the cerebral vascular endothelial expression pattern of p-ERK1/2 2 hours following DHCA and the effect of U0126 molecule at this time point. We hope the evidence provided by these studies will allow us to definitively correlate the neuronal protection achieved by the use of U0126 with the inhibition of cerebral vascular endothelial p-ERK1/2 expression.

Finally, the promising results of our initial experience with the inhibition of the ERK1/2 pathway of the MAPK signal transduction cascade by U0126 must be viewed with a global perspective of the role of the MAPK cascade in the process of cerebral I/R injury. Cerebral I/R injury not only activates the ERK1/2 subfamily of MAPK but also the p38 and JNK pathways, and the timing and magnitude of their activation within the brain are distinct. ${ }^{10}$ These MAPK cascades are not simple linear systems separately turned on or off. A complex system of cross talk exists between all 3 of these important signal transduction pathways. ${ }^{23}$ In this complex network the systematic inhibition of only 1 of these pathways, as in our study, may have unpredictable results. The problem becomes more complicated when the whole body with its multiple different tissue types (organs) is considered. Activation or inhibition of the same MAPK pathway in 2 different tissues may lead to the expression of different mediators with opposing roles. ${ }^{24}$ Thus, although these MAPK pathways represent novel targets for the therapeutic manipulation of inflammatory states and I/R injury, caution is warranted because the systemic administration of an inhibitor of a single MAPK subfamily targeting 1 organ may achieve markedly different and unintended results in other organs. ${ }^{23}$ Therefore, the overall benefit of studies such as this depend upon continued research to outline the complicated interaction between the pathways of the MAPK cascade and their effects at a tissue-specific level.

\section{Conclusion}

In summary, our findings confirm the ability of our model of DHCA to induce significant neuronal damage and activate ERK1/2 24 hours postoperatively. Furthermore, we have provided the first known report of the ability of the MEK1/ 2-specific inhibitor, U0126, to attenuate the neuronal damage associated with the use of DHCA. These results support the targeting of the MAPK cascade and the ERK1/2 pathway, in particular for inhibition as a novel therapeutic approach to mitigate the cerebral injury and neurocognitive dysfunction associated with DHCA. Future studies aimed at further elucidating the role of the MAPK cascade and the cross talk between its various pathways will help to clarify the potential role of MAPK cascade inhibitors in the treatment of cerebral I/R injury following DHCA. We hope that such experiments will eventually result in the development of a comprehensive pharmacologic neuroprotection strategy for patients undergoing DHCA.

\section{References}

1. Newburger JW, Jonas RA, Wernovsky G, et al. A comparison of the perioperative neurologic effect of hypothermic circulatory arrest versus low flow cardiopulmonary bypass in infant heart surgery. $N$ Engl J Med. 1993;329:1057-64.

2. Bellinger DC, Jonas RA, Rappaport LA, et al. Developmental and neurologic status of children after heart surgery with hypothermic circulatory arrest or low flow cardiopulmonary bypass. $N$ Engl J Med. 1995;332:549-55.

3. Hagl C, Tatton NA, Weisz DJ, Zhang N, Spielvogel D, Shiang HH, et al. Cyclosporine $\mathrm{A}$ as a potential neuroprotective agent: a study of prolonged hypothermic circulatory arrest in a chronic porcine model. Eur J Cardiothorac Surg. 2001;19:756-64.

4. Romsi P, Ronka E, Kiviluoma K, Vainionpaa V, Hirvonen J, Mennander A, et al. Potential neuroprotective benefits of erythropoietin during experimental hypothermic circulatory arrest. $J$ Thorac Cardiovasc Surg. 2002;124:714-23.

5. Langley SM, Chai PJ, Jaggers JJ, Ungerleider RM. Preoperative high dose methylprednisolone attenuates the cerebral response to deep hypothermic circulatory arrest. Eur J Cardiothorac Surg. 2000;17:279-86.

6. Rimpilainen J, Romsi P, Poekla M, Hirvonen J, Vainionpaa V, Kiviluoma KT, et al. Lamotrigine plus leukocyte filtration as a neuroprotective strategy in experimental hypothermic circulatory arrest. Ann Thorac Surg. 2002;73:163-72.

7. Ueno T, Sawa Y, Kitagawa-Sakakida S, Nishimura M, Morishita R, Kaneda $\mathrm{Y}$, et al. Nuclear factor-kB decoy attenuates neuronal damage after global brain ischemia: a future strategy for brain protection during circulatory arrest. J Thorac Cardiovasc Surg. 2001;122:720-7.

8. Davis RJ. The mitogen-activated protein kinase signal transduction pathway. J Biol Chem. 1993;268:14553-6.

9. Abe JI, Baines CP, Berk BC. Role of mitogen-activated protein kinases in ischemia and reperfusion injury: the good and the bad. Circ Res. 2000;86:607-9

10. Wu D, Ye W, Che X, Yang G. Activation of mitogen-activated protein kinases after permanent cerebral artery occlusion in mouse brain. J Cereb Blood Flow Metab. 2000;20:1320-30.

11. Barone FC, Irving EA, Ray AM, Lee JC, Kassis S, Kumar S, et al. Inhibition of p38 mitogen-activated protein kinase provides neuroprotection in cerebral focal ischemia. Med Res Rev. 2001;21:129-45. 
12. Omura T, Yoshiyama M, Shimada T, Naruhito S, Kim S, Iwao H, et al. Activation of mitogen-activated protein kinases in in-vivo ischemia/reperfused myocardium in rats. J Mol Cell Cardiol. 1999;31: 1269-79.

13. Araujo EG, Bianchi C, Faro R, Sato K, Sellke FW. Oscillation in the activities of MEK/ERK1/2 during cardiopulmonary bypass in pigs. Surgery. 2001;130:182-91.

14. Kishimoto I, Yoshmasa T, Arai H, Takaya K, Miyamoto Y, Yamashita $\mathrm{J}$, et al. Endothelin-induced activation of mitogen-activated protein kinases in glomerular mesangial cells from normotensive and strokeprone spontaneously hypertensive rats. Clin Exp Pharmacol Physiol. 1995;22(Suppl 1):S207-8.

15. Pombo CM, Bonventre JV, Avruch J, Woodgett JR, Kyriakis JM, Force T. The stress-activated protein kinases are major c-Jun aminoterminal kinases activated by ischemia and reperfusion. $J$ Biol Chem. 1994;269:26546-51.

16. Bendinelli P, Piccoletti R, Maroni P, Bernelli-Zazzera A. The MAP kinase cascades are activated during post-ischemic liver reperfusion. FEBS Lett. 1996;398:193-7.

17. Alessandrini A, Namura S, Moskowitz M, Bonventre J. MEK1 protein kinase inhibition protects against damage resulting from focal cerebral ischemia. Proc Natl Acad Sci U S A. 1999;96:12866-9.

18. Namura S, Iihara K, Takami S, Nagata I, Kikuchi H, Matsushita K, et al. Intravenous administration of MEK inhibitor U0126 affords brain protection against forebrain ischemia and focal cerebral ischemia. Proc Natl Acad Sci U S A. 2001;98:11569-74.

19. Wang H, Xu L, Venkatachalam S, Trzaskos JM, Friedman SM, Feuerstein GZ, et al. Differential regulation of IL-1beta and TNF-alpha RNA expression by MEK 1 inhibitor after focal cerebral ischemia in mice. Biochem Biophys Res Commun. 2001;286:869-74.

20. Pulsinelli WA, Brierley JB, Plum F. Temporal profile of neuronal damage in a model of transient forebrain ischemia. Ann Neurol. 1982;11:491-8.

21. Favate MF, Horiuchi KY, Manos EJ, et al. Identification of a novel inhibitor of mitogen-activated protein kinase kinase. J Biol Chem. 1998;273:18623-32.

22. DeSilva DR, Jones EA, Favata MF, Jaffee BD, Magolda RL, Trazskos $\mathrm{JM}$, et al. Inhibition of MAPK kinase blocks $\mathrm{T}$ cell proliferation but does not induce or prevent anergy. J Immunol. 1998;160:4175-81.

23. Arbabi S, Maier R. Mirogen-activated protein kinases. Crit Care Med. 2002;30:S74-9.

24. van den Blink B, Juffermans NP, ten Hove T, et al. p38 MAPK inhibition increases cytokine release by macrophages in vitro and during infection in vivo. J Immunol. 2001;166:582-7.

\section{Discussion}

Dr Carmelo A. Milano (Durham, NC). There have been some publications on the importance of administration of relatively high doses of steroids in the neonatal pig model with cardiopulmonary bypass. This has been shown to reduce fluid accumulation and also preserve end-organ function. Were steroids a part of your protocol?

Dr Mulloy. No, sir, these animals did not receive any preoperative steroids.

Dr Milano. It would be interesting then to see what simple administration of steroids would do in comparison to your inhibitor.

Dr Mulloy. Thank you for your suggestion. We will take that into consideration when planning future experiments with this model.

Dr Frank W. Sellke (Boston, Mass). MAPK is involved in a lot of different mechanisms and pathways, including vasomotion. Was there any hemodynamic effect of giving the MAPK inhibitor?

Dr Mulloy. No, sir, we did not see any significant hemodynamic effects when we gave the inhibitor. The inhibitor was given via a femoral venous line 45 minutes prior to initiation of cardio- pulmonary bypass. At this time we had an arterial line in place and the animals were attached to a pulse oximeter. Neither the blood pressure or heart rate demonstrated any significant hemodynamic change when the inhibitor was administered.

Dr Sellke. I have to commend you on your experimental preparation. I put a lot of pigs on bypass and I find it is hard to keep them alive for more than 3 hours after coming off bypass. What was the perfusion pressure after the 24 hours of recovery?

Dr Mulloy. The protocol was to keep the perfusion pressure around 40 to $50 \mathrm{~mm} \mathrm{Hg}$. At times we used small doses of dopamine, approximately 3 to $7 \mathrm{mcg} / \mathrm{kg} / \mathrm{h}$, to maintain this pressure. Overall, the average was in the 40 to 50 range.

Dr Sellke. MAPK is also involved with apoptosis. Do you think this could have had some role in your beneficial effects that you observed?

Dr Mulloy. Unfortunately we didn't use terminal deoxynucleotidyl transferase-mediated dUTP nick end labeling (TUNEL) staining as a readout in this experiment; however, we are performing some additional experiments at the moment in which we are going to include TUNEL staining as a readout. I think this will be an interesting marker and hope that we will find some beneficial effects of inhibitor administration in regard to apoptosis.

Dr Antonio F. Corno (Lausanne, Switzerland). You studied very well the control of MAPK negative effects on piglets that I presume were normoxic. We know that the vast majority of neonates requiring deep hypothermia circulatory arrest are hypoxic or cyanotic, and also we have seen MAPK implicated in the negative effects not only in ischemia and reperfusion damage but also hypoxia and reoxygenation damage, as we have demonstrated in our research lab with hypoxic myocardium. Did you study any of these protocols in hypoxic piglets, or are you planning to do it?

The second question is, what are the steps between this experimental study and the potential clinical application?

Dr Mulloy. Thank you for your comments. Unfortunately, we did not examine the role of hypoxia in this experiment. This was our first attempt to develop an appropriate model in which we could explore the effect of the MAPK cascade on cerebral ischemia/reperfusion injury, which I think is a very exciting area to explore in cardiac surgery. So we tried to stick with something fairly basic to begin; we are planning to expand from this point on. In response to the first part of your question, no, we didn't examine hypoxic animals, but your suggestion would be a nice study to do in the future.

As far as the potential steps from the bench to the bedside, I think that this work is interesting, but I think it is only the tip of the iceberg. We are looking at cerebral ischemia-reperfusion injury, and, as we all know, there are not only multiple MAPK pathways but there are multiple inflammatory cascades involved as well. However, we were excited and are encouraged that the administration of a single inhibitor was able to produce a significant decrease in neuronal damage. Nevertheless, we realize that we have a long road ahead of us and hope that future studies examining the effects of increasing the dose of the inhibitor and using inhibitors of different pathways of MAPK cascade and, as well, inhibitors of different inflammatory cascades may result in an increased beneficial effect. I think that we are still quite a distance from using this strategy at the bedside, but I think it is exciting initial work and we are happy to proceed with it. 